\title{
Transcendental Meromorphic Functions Whose First Order Derivatives Have Finitely Many Zeros
}

\author{
Feng Guo \\ Department of Mathematics, Yunnan Normal University, Kunming, China \\ Email: sdsxguofeng@163.com
}

How to cite this paper: Guo, F. (2019) Transcendental Meromorphic Functions Whose First Order Derivatives Have Finitely Many Zeros. Advances in Pure Mathematics, 9, 925-933.

https://doi.org/10.4236/apm.2019.911045

Received: October 14, 2019

Accepted: November 11, 2019

Published: November 14, 2019

Copyright $\odot 2019$ by author(s) and Scientific Research Publishing Inc. This work is licensed under the Creative Commons Attribution International License (CC BY 4.0).

http://creativecommons.org/licenses/by/4.0/

\section{cc) (i) Open Access}

\begin{abstract}
Let $f$ be a meromorphic function in $\mathbb{C}$. If the order of $f$ is greater than $2, f^{\prime}$ has finitely many zeros and $f$ takes a non-zero finite value finitely times, and then $f^{\prime}\left(f^{-1}(0)\right)$ is unbounded.
\end{abstract}

\section{Keywords}

Transcendental Meromorphic Functions, Derivatives, Zeros

\section{Introduction and Main Result}

Let $f$ be a meromorphic function in $\mathbb{C}$, define

$M_{f}=f^{\prime}\left(f^{-1}(0)\right)=\left\{f^{\prime}(z): z \in \mathbb{C}\right.$ and $\left.f(z)=0\right\}$. W. Bergweiler [1] gave a conjecture in 2001 as follow: Conjecture 1: Let $f$ be a transcendental meromorphic function in $\mathbb{C}$. If $f^{\prime}(z) \neq 1$ for all $z \in \mathbb{C}$, then $M_{f}$ is unbounded.

Bergweiler pointed that let $g(z)=z-f(z)$, Conjecture 1 is equivalent to the following one.

Conjecture 2: Let $g$ be a transcendental meromorphic function in $\mathbb{C}$. Suppose that $g^{\prime}$ does not have zeros. Then there exist a sequence $\left\{z_{n}\right\}_{n=1}^{\infty}$ of fixed points of $g$ such that $\left|g^{\prime}\left(z_{n}\right)\right| \rightarrow \infty$.

Bergweiler [1] has separately proved Conjecture 1 is affirmative for finite order meromorphic functions and entire functions; Jianming Chang [2] has confirmed the conjecture for infinite order meromorphic functions for the first time, which is based on theory of normal and quasinormal families.

For the conjecture, $f^{\prime} \neq 1$ and $f^{\prime} \neq c(c \neq 0)$ are essentially equivalent. In fact, if $f^{\prime} \neq c(c \neq 0)$, then $\frac{f^{\prime}}{c} \neq 1$ and $\frac{f}{c}$ is also transcendental meromor- 
phic function; the zeros of $f$ and the zeros of $\frac{f}{c}$ are the same and $M_{f}$ is unbounded if and only if $M_{\frac{f}{c}}$ is unbounded.

Considering the discussion above, it's natural to research the problem that whether the conclusion is true if $f^{\prime} \neq 0$ but not $f^{\prime} \neq c(c \neq 0)$, the problem is radically different to the conjecture and gives a important supplement. We can give a example to show that the problem is significant. Let $f=\mathrm{e}^{\mathrm{e}^{z}}-1$, it's obvious that $f^{\prime} \neq 0$ and $M_{f}$ is unbounded. In details, we have

Theorem 1. Let $f$ be meromorphic in $\mathbb{C}$ and the order is greater than 2. If $f^{\prime}$ has finitely many zeros and $f$ takes a finite non-zero value finitely many times, then $M_{f}$ is unbounded.

Theorem 2. Let $f$ be entire in $\mathbb{C}$ and the order is greater than 1. If $f^{\prime}$ has finitely many zeros and $f$ takes a finite non-zero value finitely many times, then $M_{f}$ is unbounded.

\section{Preliminary Lemmas}

Lemma 1. Let $f$ be a meromorphic function. If the spherical derivative $f^{\#}(z)$ of $f(z)$ is bounded. Then the order of $f(z)$ is at most 2.

For details of lemma 1, can see [3]

Remark: Let $f_{n}(z)$ be a sequence of meromorphic functions, $f_{n}(z) \stackrel{\text { loc. }}{\rightrightarrows} g(z)$ means $f_{n}(z)$ locally uniformly convergence to meromorphic function $g(z)$; for a meromorphic function $f$ in $\mathbb{C}$, let $D\left(z_{0}, M\right)=\left\{z:\left|z-z_{0}\right| \leq M\right\}$.

Lemma 2. Let $\mathcal{F}$ be a family of functions meromorphic in a domain $D$. Suppose that there exist $K>0$ such that $M_{g} \subset \overline{D(0, K)}$ for all $g \in \mathcal{F}$. For any given $\alpha$ satisfying $-1<\alpha \leq 1$, if $\mathcal{F}$ is not normal, then there exist a sequence $\left\{f_{n}\right\}_{n=1}^{\infty}$ in $\mathcal{F}$, a sequence $\left\{z_{n}\right\}_{n=1}^{\infty}$ in $D$, a sequence $\left\{\rho_{n}\right\}_{n=1}^{\infty}$ of positive real numbers and a non-constant finite order function $f$ which is meromorphic in $\mathbb{C}$ such that $z_{n} \rightarrow z_{0}$ for some $z_{0} \in D, \rho_{n} \rightarrow 0$ and

$$
\frac{f\left(z_{n}+\rho_{n} z\right)}{\rho_{n}^{\alpha}} \stackrel{\text { loc. }}{\rightrightarrows} f(z) \quad(z \in \mathbb{C} ; n \rightarrow \infty) .
$$

Moreover, the spherical derivative $f^{\#}(z)$ of $f$ satisfies $f^{\#}(z) \leq f^{\#}(0)=K+1$ for all $z \in \mathbb{C}$.

For details of lemma 2, can see [4]

In the case no hypothesis on $M_{g}$ is required, the case $\alpha=0$ is due to Zalcman [5], and the case $-1<\alpha<1$ is due to Pang [6] [7]. In the hypothesis of Lemma 2, if all $g \in \mathcal{F}$ have no zero in $D$, then $M_{g}=\varnothing$ and the conclusion is still right according to the proof of Lemma 2 in which $K$ can take 0 .

Lemma 3. Let $f$ be a meromorphic function, $D$ be an bounded domain and $c$ be constant in $\mathbb{C}$, if $f(z)-c$ has $l(l \geq 2)$ zeros in $D$ and $f^{\prime}(z)$ has $l-1$ zeros in $D$ which are all the zeros of $f(z)-c$. Then each discriminating zero of $f(z)-c$ and $f^{\prime}(z)$ in $D$ is the same one. 
Proof. Let $f(z)-c=\prod_{j=1}^{l}\left(z-z_{j}\right) g(z)=R(z) g(z)$ with $g(z)$ be a meromorphic function which have no zero in $D$ satisfying $g\left(z_{j}\right) \neq \infty(j=1, \cdots, l)$ and $R(z)=\prod_{j=1}^{l}\left(z-z_{j}\right)$ in which $z_{j} \subset D(j=1, \cdots, l)$.

Because $f^{\prime}(z)$ has $l-1$ zeros in $D$ which are all the zeros of $f(z)-c$, there exist $l-1$ points in $z_{j}(j=1, \cdots, l)$ be zeros of $f^{\prime}(z)$ and without loss of generality we may assume $f^{\prime}\left(z_{j}\right)=0(j=1, \cdots, l-1)$.

As $f^{\prime}(z)=R^{\prime}(z) g(z)+R(z) g^{\prime}(z)$, we can deduce that

$$
R^{\prime}\left(z_{j}\right)=0(j=1, \cdots, l-1), R^{\prime}(z)=l \cdot \prod_{j=1}^{l}\left(z-z_{j}\right) \text { and } \frac{R^{\prime}(z)}{R(z)}=\frac{l}{z-z_{l}}
$$

from the above it follows that $R(z)=\left(z-z_{l}\right)^{l}$ and the proof of Lemma 3 is complete.

Lemma 4. Let $f$ be a holomorphic function, if the spherical derivative of $f$ is bounded. Then the order of $f$ is at most 1.

For details of lemma 4, can see [8].

\section{Proof of Theorem 1}

Proof. we apply Lemma 1 to obtain a sequence $\left\{\omega_{n}\right\}_{n=1}^{\infty}, \omega_{n} \rightarrow \infty(n \rightarrow \infty)$ such that $f^{\#}\left(\omega_{n}\right) \rightarrow \infty,(n \rightarrow \infty) . \forall n \in \mathbb{N}$, let $f_{n}(z)=f\left(z+\omega_{n}\right)$, it's easy to apply Marty's theorem to know $\left\{f_{n}(z)\right\}_{n=1}^{\infty}$ is not normal at 0 . Suppose $f(z)-\lambda$ only have finitely many zeros $(\lambda \neq 0)$, there exist a subsequence of $\left\{f_{n}(z)\right\}_{n=1}^{\infty}$ we still suppose it's $\left\{f_{n}(z)\right\}_{n=1}^{\infty}$ such that $f(z)-\lambda$ have no zero in $\mathbb{C}$, thus according to Lemma 2 , there exist a sequence $\left\{z_{n}\right\}_{n=1}^{\infty}$, a sequence $\left\{\rho_{n}\right\}_{n=1}^{\infty}$ of positive real numbers and a non-constant finite order function $g(z)$ such that when $n \rightarrow \infty, z_{n} \rightarrow 0, \rho_{n} \rightarrow 0$ and

$$
\frac{f_{n}\left(z_{n}+\rho_{n} z\right)-\lambda}{\rho_{n}}=\frac{f\left(\omega_{n}+z_{n}+\rho_{n} z\right)-\lambda}{\rho_{n}} \stackrel{\text { loc. }}{\rightrightarrows} g(z)
$$

in $\mathbb{C}$ and $g(z)$ satisfies $g^{\#}(z) \leq g^{\#}(0)=1$ for all $z \in \mathbb{C}$.

$\forall n \in \mathbb{N}$, let $\tau_{n}=\omega_{n}+z_{n}$, there exist entire functions $F(z)$ and $H(z)$ such that $F(z)$ and $H(z)$ have no common non-trivial divisor and $f(z)=\frac{H(z)}{F(z)}$, then

$$
\begin{gathered}
\frac{f_{n}\left(z_{n}+\rho_{n} z\right)-\lambda}{\rho_{n}}=\frac{f\left(\tau_{n}+\rho_{n} z\right)-\lambda}{\rho_{n}}=\frac{\frac{H\left(\tau_{n}+\rho_{n} z\right)}{F\left(\tau_{n}+\rho_{n} z\right)}-\lambda}{\rho_{n}} \stackrel{\text { loc. }}{\rightrightarrows} g(z) \\
\frac{\rho_{n}}{f\left(\tau_{n}+\rho_{n} z\right)-\lambda} \stackrel{\text { loc. }}{\rightrightarrows} \frac{1}{g(z)}(z \in \mathbb{C})
\end{gathered}
$$


For $f\left(\tau_{n}+\rho_{n} z\right)-\lambda \neq 0$ and $g(z) \neq 0$, the derivative of (1) is

$$
\frac{\rho_{n}^{2} f^{\prime}\left(\tau_{n}+\rho_{n} z\right)}{\left\{f\left(\tau_{n}+\rho_{n} z\right)-\lambda\right\}^{2}} \stackrel{\text { loc. }}{\rightrightarrows} \frac{g^{\prime}(z)}{g^{2}(z)}(z \in \mathbb{C})
$$

here we divide two cases:

Case 1: $\frac{g^{\prime}(z)}{g^{2}(z)}$ have no zero in $\mathbb{C}$.

Because $\left(\frac{1}{g(z)}\right)^{\#}=g^{\#}(z)$ is bounded, then we apply Lemma 4 to have that the order of $\frac{1}{g(z)}$ and $\frac{g^{\prime}(z)}{g^{2}(z)}$ are at most 1. On the other hand, $\frac{g^{\prime}(z)}{g^{2}(z)} \neq 0$, we can deduce $\frac{g^{\prime}(z)}{g^{2}(z)}=\mathrm{e}^{A z+B}(A, B \in \mathbb{C}, A \neq 0)$ or constant $\alpha(\alpha \neq 0)$ and $\frac{1}{g(z)}=\frac{\mathrm{e}^{A z+B}}{A}+d \quad$ or $\frac{1}{g(z)}=\alpha z+\beta(d, \beta \in \mathbb{C})$.

here we first proof that $d \neq 0$, if $d=0$, we have

$$
\frac{f\left(\tau_{n}+\rho_{n} z\right)-\lambda}{\rho_{n}} \stackrel{\text { loc. }}{\rightrightarrows} \frac{A}{\mathrm{e}^{A z+B}}(z \in \mathbb{C})
$$

then we have that

$$
\begin{gathered}
f\left(\tau_{n}+\rho_{n} z\right)=\frac{H\left(\tau_{n}+\rho_{n} z\right)}{F\left(\tau_{n}+\rho_{n} z\right)} \stackrel{\text { loc. }}{\rightrightarrows} \lambda(z \in \mathbb{C}) \\
\frac{H\left(\tau_{n}+\rho_{n} z\right)-\lambda F\left(\tau_{n}+\rho_{n} z\right)}{F\left(\tau_{n}+\rho_{n} z\right)} \stackrel{\text { loc. }}{\rightrightarrows} 0(z \in \mathbb{C})
\end{gathered}
$$

From (4) it can be deduced that because $H\left(\tau_{n}+\rho_{n} z\right)-\lambda F\left(\tau_{n}+\rho_{n} z\right)$ have no zero, $F\left(\tau_{n}+\rho_{n} z\right)$ have no zero in any bounded domain when $n$ is large enough,. Then so are $H\left(\tau_{n}+\rho_{n} z\right)$ unite (3). What' more, $f\left(\tau_{n}+\rho_{n} z\right)$ has no zero and pole and cannot take $\lambda$ in any bounded domain, which contradict with Picard's Theorem, therefore $d \neq 0$.

From (1) we have

$$
\frac{\rho_{n}}{f\left(\tau_{n}+\rho_{n} z\right)-\lambda}-\frac{\rho_{n}}{-\lambda} \stackrel{\text { loc. }}{\rightrightarrows} \frac{1}{g(z)}(z \in \mathbb{C})
$$

Because $\frac{1}{g}$ have zero in $\mathbb{C}$, from above it can be deduced that $f\left(\tau_{n}+\rho_{n} z\right)$ have to have zeros which convergence to the zeros of $\frac{1}{g(z)}$. From (2) when $f\left(\tau_{n}+\rho_{n} z\right)=0 \quad$ then $\quad \frac{\rho_{n} f^{\prime}\left(\tau_{n}+\rho_{n} z\right)}{\lambda^{2}} \rightarrow-A d \quad$ or $\quad \alpha \quad$ and $f^{\prime}\left(\tau_{n}+\rho_{n} z\right)(n \in \mathbb{N})$ have to be unbounded.

Case 2: $\frac{g^{\prime}(z)}{g^{2}(z)}$ have zero in $\mathbb{C}$. We will proof the case is impossible. 
Take a finite zero $c$ of $\frac{g^{\prime}(z)}{g^{2}(z)}$ and it's multiple is $k(k \in \mathbb{N}, k \geq 1)$ then there exist some sufficiently small neighborhood $D_{c}$ of $c$ such that $D_{c}$ only have one zero of $\frac{g^{\prime}(z)}{g^{2}(z)}$. (2) can be expressed as

$$
\rho_{n}^{2} \cdot \frac{F^{\prime}\left(\tau_{n}+\rho_{n} z\right) H\left(\tau_{n}+\rho_{n} z\right)-H^{\prime}\left(\tau_{n}+\rho_{n} z\right) F\left(\tau_{n}+\rho_{n} z\right)}{\left\{H\left(\tau_{n}+\rho_{n} z\right)-\lambda F\left(\tau_{n}+\rho_{n} z\right)\right\}^{2}} \stackrel{\text { loc. }}{\rightrightarrows} \frac{g^{\prime}(z)}{g^{2}(z)}(z \in \mathbb{C}) .
$$

Because $f(z)$ take $\lambda$ finitely many times and from (2) $H\left(\tau_{n}+\rho_{n} z\right)-\lambda F\left(\tau_{n}+\rho_{n} z\right)$ have no zero in $D_{c}$ then when $n$ is large enough, $F^{\prime}\left(\tau_{n}+\rho_{n} z\right) H\left(\tau_{n}+\rho_{n} z\right)-H^{\prime}\left(\tau_{n}+\rho_{n} z\right) F\left(\tau_{n}+\rho_{n} z\right)$ have $k$ zeros in $D_{c}$, which are all the zeros of $F\left(\tau_{n}+\rho_{n} z\right)$ due to that

$f^{\prime}=\frac{H^{\prime}(z) F(z)-H(z) F^{\prime}(z)}{F^{2}(z)}$ only has finitely many zeros; therefore, $c$ is the zero of $\frac{1}{g(z)}$ with $k+1$ multiple and $F\left(\tau_{n}+\rho_{n} z\right)$ have $k+1$ zeros in $D_{c}$.

(1) can be expressed as

$$
\frac{\rho_{n} \frac{F\left(\tau_{n}+\rho_{n} z\right)}{H\left(\tau_{n}+\rho_{n} z\right)}}{1-\lambda \frac{F\left(\tau_{n}+\rho_{n} z\right)}{H\left(\tau_{n}+\rho_{n} z\right)}} \stackrel{\text { loc. }}{\rightrightarrows} \frac{1}{g(z)}(z \in \mathbb{C}) .
$$

From (1) we have that

$$
\lambda \frac{F\left(\tau_{n}+\rho_{n} z\right)}{H\left(\tau_{n}+\rho_{n} z\right)} \stackrel{\text { loc }}{\rightrightarrows} 1\left(z \in D_{c} \backslash\{c\}\right) .
$$

Here we divide two cases for (5):

Subcase 2.1: If $\left\{\lambda \frac{F\left(\tau_{n}+\rho_{n} z\right)}{H\left(\tau_{n}+\rho_{n} z\right)}\right\}_{n=1}^{\infty}$ is normal in $D_{c}$.

From (5) we have

$$
\begin{gathered}
\frac{\lambda F\left(\tau_{n}+\rho_{n} z\right)}{H\left(\tau_{n}+\rho_{n} z\right)} \stackrel{\text { loc }}{\rightrightarrows} 1\left(z \in D_{c}\right) . \\
\frac{\lambda F\left(\tau_{n}+\rho_{n} z\right)-H\left(\tau_{n}+\rho_{n} z\right)}{H\left(\tau_{n}+\rho_{n} z\right)} \stackrel{\text { loc. }}{\rightrightarrows} 0\left(z \in D_{c}\right) .
\end{gathered}
$$

when $n$ is large enough, notice that $H\left(\tau_{n}+\rho_{n} z\right)-\lambda F\left(\tau_{n}+\rho_{n} z\right)$ have no zero in $D_{c}$, therefore $H\left(\tau_{n}+\rho_{n} z\right)$ have no zero in $D_{c}$ and according to (6), $F\left(\tau_{n}+\rho_{n} z\right)$ have no zero in $D_{c}$ contradict with $F\left(\tau_{n}+\rho_{n} z\right)$ have $k+1$ zeros in $D_{c}$.

Subcase 2.2: If $\left\{\lambda \frac{F\left(\tau_{n}+\rho_{n} z\right)}{H\left(\tau_{n}+\rho_{n} z\right)}\right\}_{n=1}^{\infty}$ is not normal in $D_{c}$. Let

$$
\varphi_{n}(z)=\frac{\lambda F\left(\tau_{n}+\rho_{n} z\right)-H\left(\tau_{n}+\rho_{n} z\right)}{H\left(\tau_{n}+\rho_{n} z\right)} .
$$


Then $\left\{\varphi_{n}(z)\right\}_{n=1}^{\infty}$ is not normal and have no zero in $D_{c}$, we apply Lemma 2 to obtain $\left\{v_{n}\right\}_{n=1}^{\infty} \in \mathbb{C}$ and $\left\{\rho_{n}^{*}\right\}_{n=1}^{\infty}$ of positive real numbers and a non-constant finite order function $\psi(\xi)$ such that $v_{n} \rightarrow c, \rho_{n}^{*} \rightarrow 0$, and

$$
\psi_{n}(\xi)=\frac{\varphi_{n}\left(v_{n}+\rho_{n}^{*} \xi\right)}{\rho_{n}^{*}} \stackrel{\text { loc. }}{\rightrightarrows} \psi(\xi)(n \rightarrow \infty, \xi \in \mathbb{C}) \text { with } \psi^{\#}(\xi) \leq \psi^{\#}(0)=1 .
$$

here we will prove that $\psi(\xi)$ has no simple pole if it exist; let $\xi_{0}$ be the pole of $\psi(\xi)$, for $\psi(\xi)$ cannot always be $\infty$, there exist closed disc $\bar{D}\left(\xi_{0}, \delta\right)$ such that $1 / \psi(\xi)$ and $1 / \psi_{n}(\xi)$ are holomorphic in $\bar{D}\left(\xi_{0}, \delta\right)$ and $1 / \psi_{n}(\xi) \rightrightarrows 1 / \psi(\xi)$ uniformly in $\bar{D}\left(\xi_{0}, \delta\right)$ and so are $1 / \psi_{n}(\xi)+\rho_{n}^{*}$.

Notice that $1 / \psi_{n}(\xi)$ cannot be constant, there exist $\left\{\xi_{n}\right\}_{n=1}^{\infty}, \xi_{n} \rightarrow \xi_{0}(n \rightarrow \infty)$ such that

$$
\frac{1}{\psi_{n}\left(\xi_{n}\right)}+\rho_{n}^{*}=\frac{\rho_{n}^{*}}{\varphi_{n}\left(v_{n}+\rho_{n}^{*} \xi_{n}\right)}+\rho_{n}^{*}=0, \quad \varphi_{n}\left(v_{n}+\rho_{n}^{*} \xi_{n}\right)+1=0 .
$$

We firstly show that the discriminating zeros of $\varphi_{n}(z)+1$ in $D_{c}$ are all the zeros of $\varphi_{n}^{\prime}(z)$ in $D_{c}$ when $n$ is large enough. In fact, we have that the $k$ zeros of $F^{\prime}\left(\tau_{n}+\rho_{n} z\right) H\left(\tau_{n}+\rho_{n} z\right)-H^{\prime}\left(\tau_{n}+\rho_{n} z\right) F\left(\tau_{n}+\rho_{n} z\right)$ as same as $\varphi_{n}^{\prime}(z)$, which are all belong to the $k+1$ zeros of $F\left(\tau_{n}+\rho_{n} z\right)$ and $\varphi_{n}(z)+1$ in $D_{c}$, then Lemma 3 can be used to prove the conclusion and we further have

$$
\left.\left(\frac{1}{\psi(\xi)}\right)^{\prime}\right|_{\xi=\xi_{0}}=-\frac{\psi^{\prime}\left(\xi_{0}\right)}{\left\{\psi\left(\xi_{0}\right)\right\}^{2}}=-\lim _{n \rightarrow \infty} \frac{\psi_{n}^{\prime}\left(\xi_{n}\right)}{\left\{\psi_{n}\left(\xi_{n}\right)\right\}^{2}}=-\lim _{n \rightarrow \infty}\left\{\rho_{n}^{*}\right\}^{2} \cdot 0=0
$$

which means $\psi(\xi)$ has to have multiple pole if it exist.

$$
\text { Notice } \frac{H^{\prime}(z) F(z)-H(z) F^{\prime}(z)}{F^{2}(z)} \text { only has finitely many zeros, then }
$$
$H\left(\tau_{n}+\rho_{n} z\right)$ have no multiple zero in $\mathbb{C}$ when $n$ is large enough.

Considering $1 / \psi_{n}(\xi) \stackrel{\text { loc. }}{\rightrightarrows} 1 / \psi(\xi)(\xi \in \mathbb{C})$, since $\lambda F\left(\tau_{n}+\rho_{n} z\right)-H\left(\tau_{n}+\rho_{n} z\right)$ have no zero in $D_{c}$ when $n$ is large enough, $1 / \psi_{n}(\xi)$ are analytic in $D_{c}$ and according to Hurwitz's Theorem, $\psi(\xi)$ has no multiple pole. With the assert above, $\psi(\xi)$ have no pole and be entire.

Notice that $\psi^{\#}(\xi) \leq \psi^{\#}(0)=1(\xi \in \mathbb{C})$ and Lemma 4 , the order of $\psi(\xi)$ is at most 1 . Since $\varphi_{n}(z)$ have no zero in $D_{c}$ when $n$ is large enough, then $\varphi_{n}\left(v_{n}+\rho_{n}^{*} \xi\right)$ have no zero in any bounded domain, from the above it follows that $\psi(\xi) \neq 0(\xi \in \mathbb{C})$ and $\psi(\xi)=\mathrm{e}^{A \xi+B}(A, B \in \mathbb{C}, A \neq 0)$.

$$
\begin{aligned}
& 1 / \psi_{n}(\xi) \stackrel{\text { loc. }}{\rightrightarrows} 1 / \psi(\xi)(\xi \in \mathbb{C}) \text { is } \\
& \frac{\rho_{n}^{*} H\left(\tau_{n}+\rho_{n} v_{n}+\rho_{n} \rho_{n}^{*} \xi\right)}{\lambda F\left(\tau_{n}+\rho_{n} v_{n}+\rho_{n} \rho_{n}^{*} \xi\right)-H\left(\tau_{n}+\rho_{n} v_{n}+\rho_{n} \rho_{n}^{*} \xi\right)} \stackrel{\text { loc. }}{\rightrightarrows} \mathrm{e}^{-A \xi-B} \quad(\xi \in \mathbb{C}) .
\end{aligned}
$$

Let

$$
\eta_{n}(z)=\frac{F\left(\tau_{n}+\rho_{n} z\right) H^{\prime}\left(\tau_{n}+\rho_{n} z\right)-F^{\prime}\left(\tau_{n}+\rho_{n} z\right) H\left(\tau_{n}+\rho_{n} z\right)}{\left\{\lambda F\left(\tau_{n}+\rho_{n} z\right)-H\left(\tau_{n}+\rho_{n} z\right)\right\}^{2}}
$$


then the derivative of (7) is

$$
\left(\rho_{n}^{*}\right)^{2} \rho_{n} \lambda \eta_{n}\left(v_{n}+\rho_{n}^{*} \xi\right) \stackrel{l o c .}{\rightrightarrows}-A \mathrm{e}^{-A \xi-B}(n \rightarrow \infty, \xi \in \mathbb{C}) .
$$

(2) can be expressed as

$$
\rho_{n}^{2} \eta_{n}(z) \stackrel{\text { loc. }}{\rightrightarrows} \frac{g^{\prime}(z)}{g^{2}(z)}(n \rightarrow \infty, z \in \mathbb{C}) .
$$

$\forall n \in \mathbb{N}$, let $h_{n}(z)$ be the $k$ order derivative of $\eta_{n}(z)$, then the $k$ order derivative of (2) is

$$
\rho_{n}^{2} h_{n}(z) \stackrel{\text { loc. }}{\rightrightarrows}\left(\frac{1}{g(z)}\right)^{(k+1)}(n \rightarrow \infty, z \in \mathbb{C})
$$

with $\left(\frac{1}{g(z)}\right)^{(k+1)}$ have no zero in $D_{c}$. Let $\left.\left(\frac{1}{g(z)}\right)^{(k+1)}\right|_{z=c}=G_{c}(\neq 0)$ then we have

$$
\rho_{n}^{2} h_{n}\left(v_{n}+\rho_{n}^{*} \xi\right) \stackrel{\text { loc. }}{\rightrightarrows} G_{c}(n \rightarrow \infty, \xi \in \mathbb{C}) .
$$

The $k$ order derivative of $(8)$ is

$$
\left(\rho_{n}^{*}\right)^{k+2} \rho_{n} \lambda h_{n}\left(v_{n}+\rho_{n}^{*} \xi\right) \stackrel{\text { loc. }}{\rightrightarrows}(-A)^{k+1} \mathrm{e}^{-A \xi-B} \quad(n \rightarrow \infty, \xi \in \mathbb{C}),
$$

$(9)+(10)$ is

$$
\left\{\rho_{n}+\lambda\left(\rho_{n}^{*}\right)^{k+2}\right\} \rho_{n} h_{n}\left(v_{n}+\rho_{n}^{*} \xi\right) \stackrel{l o c .}{\rightrightarrows} G_{c}+(-A)^{k+1} \mathrm{e}^{-A \xi-B}(n \rightarrow \infty, \xi \in \mathbb{C}) .
$$

It shows that $h_{n}\left(v_{n}+\rho_{n}^{*} \xi\right)$ have to have zeros in $\mathbb{C}$ when $n$ is large enough, however, from (10) and Hurwitz's theorem, it is impossible; this gives a contradiction and the proof of Theorem 1 is complete.

\section{Remarks}

It follows from the proof of Theorem 1 that the hypothesis for order can be replaced by greater than 1 for entire functions. In fact, from Lemma 4, we can obtain a sequence $\left\{\omega_{n}\right\}_{n=1}^{\infty}, \omega_{n} \rightarrow \infty(n \rightarrow \infty)$ such that $f^{\#}\left(\omega_{n}\right) \rightarrow \infty,(n \rightarrow \infty)$. Then using the start point of proof of Theorem $1, \forall n \in \mathbb{N}$, let $f_{n}(z)=f\left(z+\omega_{n}\right)$, it's easy to apply Marty's theorem to know $\left\{f_{n}(z)\right\}_{n=1}^{\infty}$ is not normal at 0 . Suppose $f(z)-\lambda$ only has finitely many zeros $(\lambda \neq 0)$, there exist a subsequence of $\left\{f_{n}(z)\right\}_{n=1}^{\infty}$. We still suppose it's $\left\{f_{n}(z)\right\}_{n=1}^{\infty}$ such that $f_{n}(z)-\lambda$ has no zero in $\mathbb{C}$. Thus according to Lemma 2 , there exist a sequence $\left\{z_{n}\right\}_{n=1}^{\infty}$, a sequence $\left\{\rho_{n}\right\}_{n=1}^{\infty}$ of positive real numbers and a non-constant finite order function $g(z)$ such that when $n \rightarrow \infty$, $z_{n} \rightarrow 0, \rho_{n} \rightarrow 0$ and

$$
f_{n}\left(z_{n}+\rho_{n} z\right)-\lambda=f\left(\omega_{n}+z_{n}+\rho_{n} z\right)-\lambda \stackrel{\text { loc. }}{\rightrightarrows} g(z)
$$

in $\mathbb{C}$ and $g(z)$ satisfies $g^{\#}(z) \leq g^{\#}(0)=1$ for all $z \in \mathbb{C}$. For $g^{\prime} \neq 0$ and $g \neq 0$ we apply Lemma 6 to have the order of $g$ at most 1 , and 
$g=\mathrm{e}^{A z+B}(A \neq 0)$ and we have

$$
f\left(\omega_{n}+z_{n}+\rho_{n} z\right) \stackrel{l o c}{\rightrightarrows} \mathrm{e}^{A z+B}+\lambda(n \rightarrow \infty, z \in \mathbb{C}) .
$$

and the first order derivative is

$$
\rho_{n} f^{\prime}\left(\omega_{n}+z_{n}+\rho_{n} z\right) \stackrel{l o c .}{\rightrightarrows} A \mathrm{e}^{A z+B}(n \rightarrow \infty, z \in \mathbb{C}) .
$$

If $f\left(\omega_{n}+z_{n}+\rho_{n} z\right)=0 \quad$, then $\rho_{n} f^{\prime}\left(\omega_{n}+z_{n}+\rho_{n} z\right) \rightarrow-A \lambda \quad$ and $f^{\prime}\left(\omega_{n}+z_{n}+\rho_{n} z\right)(n \in \mathbb{N})$ is unbounded. Then the proof of Theorem 2 is complete.

The requirement for order in Theorem 2 is sharp, let $f=\mathrm{e}^{z}-1$, then $M_{f}=\{1\}$.

By the equivalence between the conjecture 1 and 2, we can have two corollaries from Theorem 1 and 2 .

Corollary 1. Let $g$ be meromorphic in $\mathbb{C}$ and the order is greater than 2. If $g^{\prime}-1$ has finitely many zeros and $g-z$ takes a finite non-zero value finitely many times, then $g$ has a sequence $\left\{z_{n}\right\}_{n=1}^{\infty}$ of fixed points such that $g^{\prime}\left(z_{n}\right) \rightarrow \infty,(n \rightarrow \infty)$.

Corollary 2. Let $g$ be entire in $\mathbb{C}$ and the order is greater than 1 . If $g^{\prime}-1$ has finitely many zeros and $g-z$ takes a finite non-zero value finitely many times, then $g$ has a sequence $\left\{z_{n}\right\}_{n=1}^{\infty}$ of fixed points such that $g^{\prime}\left(z_{n}\right) \rightarrow \infty,(n \rightarrow \infty)$.

\section{Acknowledgements}

I thank the Editor and the referee for their comments. Research of F. Guo is funded by the Yunnan province Science Foundation grant 2016FD015. This support is greatly appreciated.

\section{Conflicts of Interest}

The author declares no conflicts of interest regarding the publication of this paper.

\section{References}

[1] Bergweiler, W. (2001) Normality and Exceptional Values of Derivatives. Proceedings of the American Mathematical Society, 129, 121-129. https://doi.org/10.1090/S0002-9939-00-05477-0

[2] Chang, J.M. (2012) On Meromorphic Functions Whose First Derivatives Have Finitely Many Zeros. Bulletin of the London Mathematical Society, 44, 703-715. https://doi.org/10.1112/blms/bds003

[3] Ahlfors, L. (1929) Beiträge zur Theorie der Meromorphen Funktionen. C.R.T Congr.Math., Scand.Oslo, 19, 84-88.

[4] Pang, X.C. and Zalcman, L. (2000) Normal Families and Shared Values. Bulletin of the London Mathematical Society, 32, 325-331. https://doi.org/10.1112/S002460939900644X

[5] Zaclman, L. (1975) A Heuristic Principle in Complex Function Theory. The Amer- 
ican Mathematical Monthly, 82, 813-817.

https://doi.org/10.1080/00029890.1975.11993942

[6] Pang, X.C. (1989) Bloch's Principle and Normal Criterion. Science in China Series $A, 32,782-791$.

[7] Pang, X.C. (1990) On Normal Criterion of Meromorphic Functions. Science in China Series A, 33, 521-527. https://doi.org/10.1360/ya1990-33-5-521

[8] Clunie, J. and Hayman, W.K. (1966) The Spherical Derivative of Integral and Meromorphic Functions. Commentarii Mathematici Helvetici, 40, 117-148.

https://doi.org/10.1007/BF02564366 